\title{
Supernova Tsunami: Cosmic Core-Collapse Explosions in Upcoming Sky Surveys
}

\author{
Amy Lien* \\ University of Illinois at Urbana-Champaign \\ E-mail: amylien2@uiuc.edu \\ Brian Fields \\ University of Illinois at Urbana-Champaign
}

\begin{abstract}
Our observational understanding of supernovae of all types, and core-collapse supernovae in particular, will soon experience a revolution due to the flood of new data from scanning (synoptic) sky surveys. In the coming decade, many such surveys will begin, including DES, PanSTARRS, LSST; indeed SDSS-II is already ongoing. We quantify the discovery potential of the core-collapse supernovae of these surveys. The number and redshift depth of the supernovae discovered strongly depend on the survey limiting magnitude. With the limiting magnitude suggested by these surveys, from $\sim 10^{3}$ to eventually $\sim 10^{5}$ supernovae will be discovered per year, out to redshift $\mathrm{z} \sim 1$. This catalog will rapidly dwarf the present $\sim 4800$ events in recorded history starting one millennium ago with SN1006. We will present detailed forecasts for the huge supernova harvest which will result from these surveys. We will go on to discuss the dramatic impact on supernova science as well as cosmology. Applications include: (1) High statistics measurements of light curves for all supernova types sampled in an unbiased way across galaxy types, which will promise a better understanding of the observable properties of the core-collapse supernovae and their evolution with environment and redshift out to redshift $z \sim 1$. (2) A more precise estimate of the cosmic supernova rate and star formation rate. (3) The use of core-collapse supernovae as distance indicators via the expanding photosphere method. The surveys will provide an independent cross-check on the distance measurements from Type Ia supernovae. (4) Calibrated predictions of the supernova neutrino background.
\end{abstract}

10th Symposium on Nuclei in the Cosmos

July 27 - August 12008

Mackinac Island, Michigan, USA

\footnotetext{
* Speaker.
} 


\section{Introduction}

This paper is the summary of the work of Lien \& Fields (2008), which focuses on the ubiquitousness of the core-collapse supernovae $(\mathrm{CC} \mathrm{SNe})$ and their scientific potential. As mentioned in Woosley \& Janka (2005), CC SNe are important for many aspects of cosmology and astrophysics generally, and are central actors in nuclear astrophysics. For example, CC SNe result from the violent explosion of short-lived massive stars, hence CC SN rate is closely related to star formation rate (Madau et al. 1996). Furthermore, metals are created during different stages of nuclear fusion in massive stars and are released into the environment via the explosions of supernovae. Therefore $\mathrm{CC} \mathrm{SNe}$ are the dominant source of galactic and intergalactic metals. In additional to this, $\mathrm{CC} \mathrm{SNe}$ are also an important source of neutrinos. About $99 \%$ of the energy released from supernovae is in the form of neutrinos and antineutrinos of all species with energy from $\sim 10 \mathrm{MeV}$ to $\sim 30 \mathrm{MeV}$.

The following decade will see several deep-field surveys with large scanning areas, including DES, LSST, Pan-STARRS, and the already ongoing SDSS-II. These surveys will detect $\gtrsim 100,000$ supernovae annually up to redshift $\mathrm{z} \sim 1$, which is several orders of magnitude greater than the number observed until now. We summarize the main features of these surveys in the first three columns of Table 1. Note that the limiting magnitude we used for CC SNe is 1 magnitude less than the limiting magnitude proposed by each survey mainly in order to measure the light curve and thus infer supernova type.

Table 1: Current and Future Synoptic Sky Surveys and Their Discovery Potential in $r$-band.

\begin{tabular}{c|ccccc}
\hline \hline Survey & $\begin{array}{c}\text { Scan Area } \\
\text { Name }\end{array}$ & $\begin{array}{c}\text { SN Depth } \\
\Omega_{\text {scan }}\left[\mathrm{deg}^{2}\right]\end{array}$ & $\begin{array}{c}\text { Expected } \\
m_{\text {lim }}^{\text {sn }}[\mathrm{mag}]\end{array}$ & $\begin{array}{c}\text { Expected Total 1-year } \\
\text { Operation }\end{array}$ & $\begin{array}{c}\text { SNII Redshift } \\
\text { SNII Detections }\end{array}$ \\
\hline SDSS-II & 300 & 21.5 & $2005-2008$ & $1.70 \times 10^{2}$ & $0.03<z<0.37$ \\
DES & 40 & 24.2 & $2011-2016$ & $2.74 \times 10^{3}$ & $0.06<z<1.20$ \\
Pan-STARRS & 30000 & 23 & $2010-2020$ & $5.14 \times 10^{5}$ & $0.01<z<0.89$ \\
LSST & 20000 & $23-25$ & $2014-2024$ & $3.43 \times 10^{5}$ & $0.01<z<0.89$ \\
\hline \hline
\end{tabular}

Note: ${ }^{*}$ Reflects SDSS-II supernova scan season of 3 months per calendar year.

Survey design and forecasts to date have focused on Type Ia supernovae because of their obvious importance in cosmology. Type IIn explosions can be as bright or brighter than Type Ia explosions, but more common CC SN types are typically dimmer by one magnitude or more. Nevertheless, future surveys will detect enormous numbers of CC SNe which will come for free. Moreover, CC SN detection has basically the same revisit timescale ("cadence"), about 4 days.

\section{Method}

Lien \& Fields (2008) estimates the detection of CC SNe supernovae in upcoming surveys. Our procedure is similar to those described in Dahlén \& Fransson(1999).

The ideal cosmic CC SNe rate can be related to cosmic star formation rate by the fraction of massive stars, found using the initial mass function $\xi(m)$,

$$
\mathscr{R}_{\mathrm{SN}}=\frac{\int_{8 M_{\odot}}^{100 M_{\odot}} \xi(m) d m}{\int_{0.1 M_{\odot}}^{100 M_{\odot}} m \xi(m) d m} \dot{\rho}_{\star}=\frac{0.00965}{M_{\odot}} \dot{\rho}_{\star}
$$



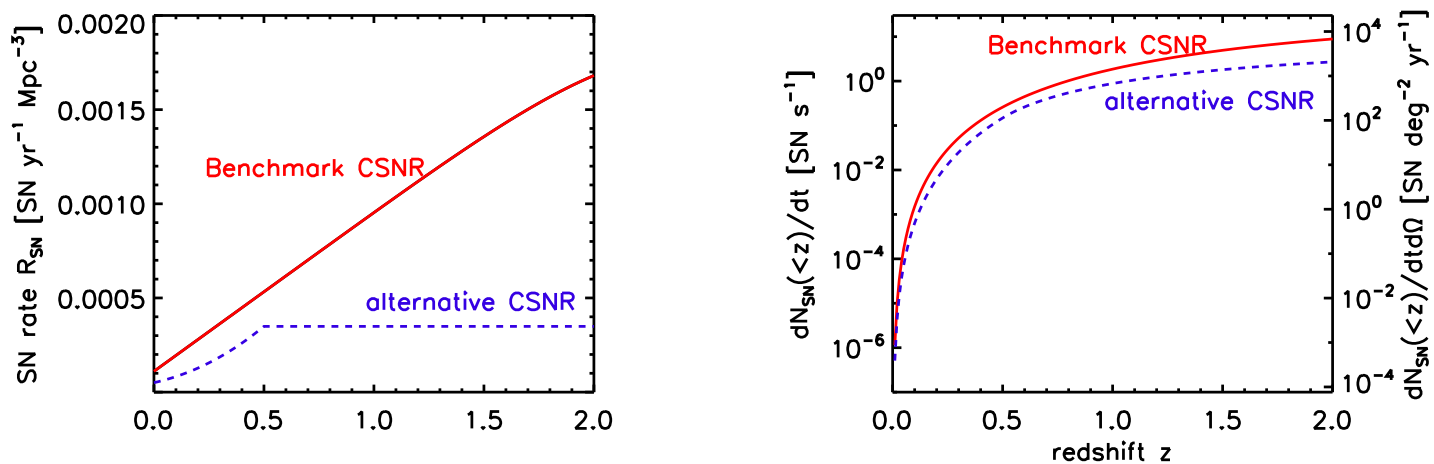

Figure 1: (a) Left panel: Possible cosmic core-collapse supernova rates as a function of redshift. The solid curve is the result calculated based on the fiducial "benchmark" cosmic star-formation rate; the broken curve is the alternative cosmic supernova rate we used for comparison. (b) Right panel: The idealized, all-sky cumulative rate of all supernovae observed over redshift 0 to $z$, for an observer with no faintness limit and with no dust extinction anywhere along the line of sight.

Our "benchmark" cosmic CC SNe rate (CSNR) is calculated from the Cole et al. (2001) star formation rate with parameters fitted by Hopkins \& Beacom (2006) according to current observational data. In order to see how different choices of CSNR would affects the results, we also do all our calculations with an alternative CSNR, which is based on the current CC SNe observational data. Figure 1 shows the result of the two CSNRs we used. The results show that one supernova explodes every second in the universe out to redshift $z \sim 1$.

Although the all-sky CC SN rate is huge, not all of them are observable due to host galaxy dust obscuration and survey limiting magnitude. In order to estimate the number of supernovae detected by each survey, dust extinction and the detection limit of each instrument must be considered.

A major impediment to supernova discovery, and probably the largest uncertainty in our estimates, arises when supernovae are rendered invisible due to extinction by dust in their host galaxies. Mannucci et al. (2007) estimated the missing fraction $\alpha$ of CC SNe due to dust obscuration. We adopted this estimate and get the observable fraction $f_{\text {dust }}=1-\alpha$ due to dust. We also consider extinction and reddening to the observable supernovae (Richardson et al. 2002).

The fraction $f_{\text {maglim }}$ of supernovae brighter than the detection limit is calculated with the luminosity functions for different types of CC SNe (Ib, Ic, IIL, IIp, and IIn) provided by Richardson et al. (2002). The $K$-correction is also considered when estimating $f_{\text {maglim }}$ and we adopted the SDSS filter with the AB magnitude system (The SDSS Collaboration(2006)).

Then the total detectable fraction of CC SNe in the filter band $x$ would be

$$
f_{\text {detect }, \mathrm{x}}\left(z ; m_{\mathrm{lim}}^{\mathrm{sn}}\right)=f_{\text {maglim }, \mathrm{x}}\left(z ; m_{\mathrm{lim}}^{\mathrm{sn}}\right) f_{\text {dust }}(z)
$$

Finally, the detectable cosmic CC SNe rate in filter band $x$ would just be the ideal cosmic CC SNe rate times the detectable fraction $f_{\text {detect, }}\left(z ; m_{\text {lim }}^{\text {sn }}\right)$. 


\section{Results}

Our estimations for the total number of $\mathrm{CC} \mathrm{SNe} \mathrm{detections} \mathrm{and} \mathrm{the} \mathrm{corresponding} \mathrm{redshift}$ ranges for the future surveys are summarized in the last two columns of Table 1.

Figure 2 shows our general results in $r$-band. The left panel shows the observed cosmic CC $\mathrm{SNe}$ rate for different $m_{\mathrm{lim}}^{\mathrm{sn}}$ in $r$-band. The right panel shows the estimates for the LSST survey for different $m_{\mathrm{lim}}^{\mathrm{sn}}$. The upper plot of the right panel shows the number of supernova detections per year with different survey limiting magnitudes and the lower plot shows the time needed to achieve a $10 \%$ precision for the CC SNe rate.
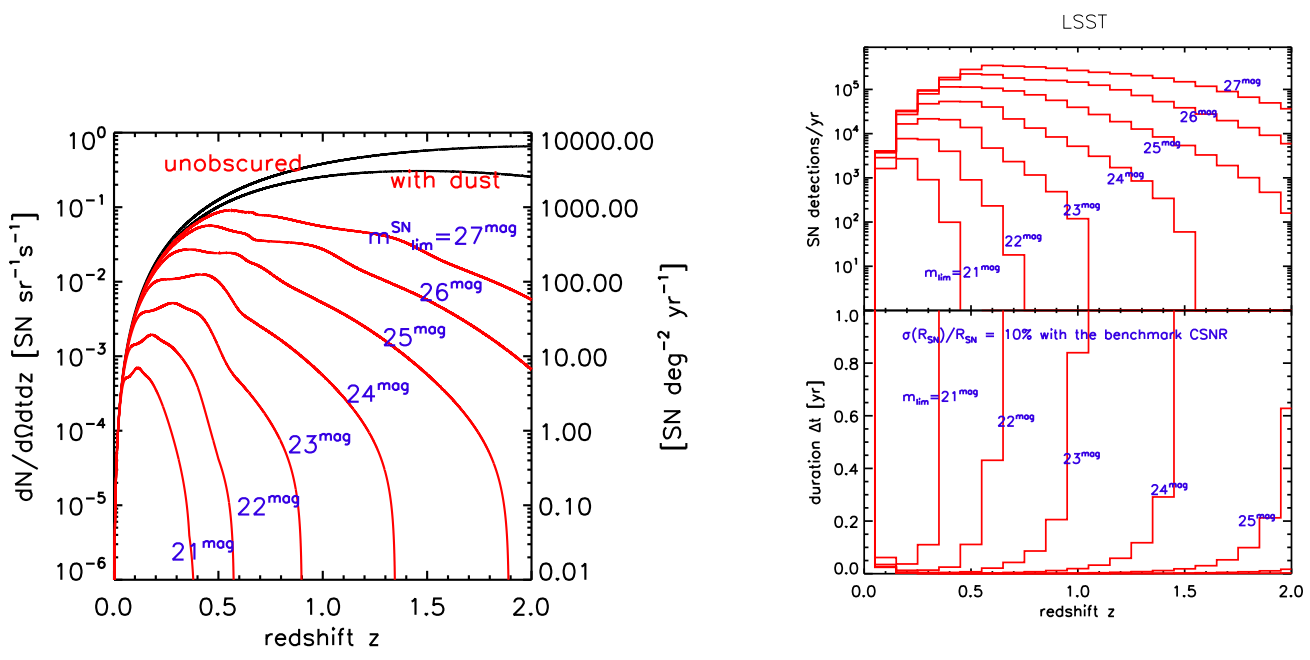

Figure 2: The left panel: the observing CC SNe rate with different survey limit magnitudes. Also showing the ideal (unobscured) rate and the rate with dust effect only for comparison. The right panel: The upper plot shows the number of supernovae observed in one year, in redshift bins of width $\Delta z=0.1$. Results are shown for a fixed scan sky coverage $\Delta \Omega_{\text {scan }}=20000 \mathrm{deg}^{2}$, and the survey depth as labeled. The lower plot shows the observing time $\Delta t$ needed in order to determine the cosmic supernova rate to a $10 \%$ precision.

Figure 2 and the last two columns of Table 1 show that synoptic surveys should see enormous numbers of supernovae up to redshift $\mathrm{z} \sim 1$. Also, the number and the redshift range of the observed CC SNe both increase rapidly with $m_{\mathrm{lim}}^{\mathrm{sn}}$. The lower plot of the right panel of Fig. 2 also shows that with the suggested limiting magnitude $m_{\lim }^{\mathrm{sn}}=23$, LSST can easily measure the cosmic CC SNe rate via direct counting to a $10 \%$ precision out to redshift $z \sim 1$.

\section{Conclusion}

In the next decade, the upcoming surveys will reap a huge harvest of CC SNe. For example, LSST will detect $\sim 10^{5} \mathrm{CC} \mathrm{SNe}$ per year out to redshift $z \sim 1$ with the limiting magnitude suggested by the survey $\left(m_{\mathrm{lim}}^{\mathrm{sn}}=23\right)$. This unprecedented flood of CC SNe data can be brought to bear on a wide range of applications. Statistical analysis could be applied to supernova science, such as supernova light curves, luminosity functions, environmental dependence, etc. Furthermore, measuring the CSNR from direct counting can be achieved to high precision and provide an alternative estimate of the star formation rate. Also, it is possible to use CC SNe as distance indicators via 
the method of expanding photospheres, which has received intensified interest recently. Therefore CC SNe might provide an independent cross check for the distance measurements from Type Ia supernovae.

Follow-up spectroscopy will be needed for some of the applications listed above. However, this will yield a huge scientific payoff, which makes it worthwhile to consider doing follow-up on some selective CC SNe.

Our results also show that the limiting magnitude $m_{\lim }^{\text {sn }}$ plays an crucial role when determining the detectable CC SNe number and the redshift range. There will be a large payoff when increasing one magnitude in $m_{\mathrm{lim}}^{\mathrm{sn}}$. For example, $m_{\mathrm{lim}}^{\mathrm{sn}}=25$ will increase the number of detections by an order of magnitude and put the detection redshift range to $z \sim 2$. Of course, increasing $m_{\lim }^{\mathrm{sn}}$ will mean a certain amount of trade-off from the cadence and possibly also from the scanning area. However, one might be able to find a certain $m_{\mathrm{lim}}^{\mathrm{sn}}$ which maximize the benefit in the interested field.

In closing, we reiterate that upcoming surveys tuned to observe Type Ia supernovae will automatically discover a huge number of CC SNe. Exploiting these data will offer new insights into nuclear astrophysics, cosmology, and supernova science.

Acknowledgments. We would like to thank Hendrik Schatz, Rich Cyburt and all of the organizers for a very stimulating meeting in a spectacular setting. A.L. is grateful to the organizers for support which made her attendance possible.

\section{References}

[1] Cole, S., et al. 2001, MNRAS, 326, 255

[2] Dahlén, T., \& Fransson, C. 1999, A\&A, 350, 349

[3] Hopkins, A. M., \& Beacom, J. F. 2006, ApJ, 651, 142

[4] Lien, A., \& Fields, B. 2008, submitted to JCAP

[5] Madau, P., Ferguson, H. C., Dickinson, M. E., Giavalisco, M., Steidel, C. C., \& Fruchter, A. 1996, MNRAS, 283, 1388

[6] Mannucci, F., Della Valle, M., \& Panagia, N. 2007, MNRAS, 377, 1229

[7] Richardson, D., Branch, D., Casebeer, D., Millard, J., Thomas, R. C., \& Baron, E. 2002, AJ, 123, 745

[8] Sloan Digital Sky Survey Collaboration http://sdssdp47.fnal.gov/sdsssn/sdsssn.html

[9] Woosley, S., \& Janka, T. 2005, Nature Physics, 1, 147 